\title{
Consejeria en alimentación, actividad física y tabaco. Instrumento fundamental en la practica profesional
}

\section{Counseling in feeding, physical activity and tobacco. Basic instrument in professional practice}

\begin{abstract}
The aim of this article was to present an update of the theorical and methodological foundations of counseling and evidence of their effectiveness, relating to the practice of health teams and analyzing future challenges. It is described, the theorical basis, the policy directions of the Chilean Ministry of Health, the international recommended strategies and the contributions of the participants in a training program conducted by INTA with 312 professionals (nutritionists, physiotherapists and dentists mostly). The analysis of the conditions in the Primary Health Care, to implement systematically nutrition, physical activity and tobacco counseling is presented. The need to improve the practice and its contribution to a preventive approach to serious problems of physical inactivity, obesity and smoking in the Chilean population is discussed.

Key words: counseling in feeding, physical activity, tobacco, stages of change, motivational interviewing, $5 A$ and $5 R$ strategy.
\end{abstract}

\section{INTRODUCCION}

Los rápidos cambios epidemiológicos y nutricionales de los últimos años han significado que las Enfermedades Crónicas No Transmisibles (ECNT) han pasado a constituir la principal causa de enfermedad y muerte en Chile y en el mundo. Un grupo importante de estas enfermedades dependen de la alimentación, de la actividad física y del consumo de tabaco, junto a determinantes sociales de la salud, (1).

Una de las estrategias recomendada para el abordaje de esta problemática es la consejería. Existe consenso sobre la eficacia de la consejería efectuada por profesionales de salud, cuando se realiza en forma sistemática y bajo ciertos criterios de calidad, entre los cuales está el uso de adecuadas metodologías de comunicación interpersonal, (2).

Ante la necesidad de desarrollar competencias y habilidades del personal de salud, el Instituto de Nutrición y Tecnología de los Alimentos (INTA) de la Universidad de Chile, ha impartido programas de formación para equipos de Atención
Judith Salinas C. (1)

Sergio Bello S. $(1,2)$

Haydee Chamorro R. (2)

Carmen Gloria Gonzalez G. (1)

(1) Instituto de Nutricion y Tecnologia de los Alimentos INTA, Universidad de Chile, Santiago, Chile.

(2) Instituto Nacional del Tórax, Santiago, Chile.

Dirigir la correspondencia a: Profesora Judith Salinas Instituto de Nutrición y Tecnología de los Alimentos (INTA) Universidad de Chile. El Líbano 5524, Casilla 138, Correo 11 Santiago, Chile. Fax: 56-2 2214030 e-mail: jsalinas@inta.uchile.cl Este trabajo fue recibido el 22 de Julio de 2016
y aceptado para ser publicado el 10 de Noviembre de 2016 .

Primaria de Salud (APS) (3), en consejería de alimentación, actividad física y tabaco, orientada a apoyar la adquisición de estilos de vida saludable y contribuir a la disminución de los problemas de sobrepeso, obesidad y tabaquismo. En el período académico 2014 - 2015 se realizan 12 cursos a 312 profesionales de diferentes regiones del país, con un promedio de 26 estudiantes por curso: 60\% nutricionistas, kinesiólogos y odontólogos, y el 40\% restante de otras profesiones (cuadro 1).

El proceso de aprendizaje es teórico-práctico, de carácter presencial con metodología participativa e interactiva, casos de estudio, juego de roles y videos de intervenciones de comunicación centrada en la persona. En el inicio de cada programa, los estudiantes analizan la experiencia práctica de consejería realizada durante el último año previo al curso. Posteriormente, una vez finalizada la capacitación identifican las oportunidades que tiene la APS para una práctica sistemática y efectiva, planteando recomendaciones.

El objetivo de este artículo fue actualizar los fundamentos 
de la consejería, su evidencia y las metodologías de implementación, relacionándolo con las políticas y la práctica observada en los equipos de salud.

La consejería en las políticas de salud

Durante el año 2003 el INTA, en convenio con el MINSAL, elaboró Guías y Manuales para realizar Consejería en Vida Sana en el marco del Plan Nacional de Promoción de Salud $(4,5)$. Estos materiales educativos se distribuyeron a todos los Servicios de Salud y establecimientos de APS con la recomendación de su uso, quedando la formación de los equipos y apoyo para su implementación a iniciativa local, $(6,7)$.

El Ministerio de Salud (MINSAL) ha incluido la recomendación de realizar consejería en diferentes programas de salud, en especial de salud cardiovascular, de la mujer y del adulto mayor (8-12). A partir del 2006 los distintos tipos de conseje- ría, son parte del sistema de registro de actividades mensuales que debe realizar el personal de salud (Resúmenes Estadísticos Mensuales-REM); el que incluye consejerías de promoción de salud, prevención de algunas enfermedades y de apoyo a tratamientos (13). Los fundamentos de la consejería están planteados en las Orientaciones del Modelo de Salud Familiar (14) y en la Programación anual de la Red Asistencial (15). Es un factor de certificación de los Centros de Salud Familiar (16), con la recomendación de realizar consejería con adolescentes, mujeres, adultos y adultos mayores, en actividad física, tabaquismo y alimentación saludable. Sin embargo, ninguno de estas orientaciones se acompaña de protocolos que permitan una implementación de calidad a nivel país.

El Examen de Medicina Preventiva del Adulto (EMPA) anual, es la única prestación de salud garantizada a toda la población beneficiaria del sistema de salud público y privado

\section{TABLA 1}

Consejería en Alimentación, Actividad Física y Tabaco. Participantes de cursos de perfeccionamiento según profesión. INTA Universidad de Chile 2014-2015.

\begin{tabular}{|c|c|c|c|}
\hline & $\begin{array}{c}\text { Consejería Alimentación } \\
\text { y Actividad Física }\end{array}$ & Consejería Tabaco & Total \\
\hline Nutricionista & 44 & 23 & 67 \\
\hline Kinesiólogo & 24 & 53 & 77 \\
\hline Odontólogo & 02 & 48 & 50 \\
\hline Enfermera & 14 & 13 & 27 \\
\hline Psicólogo & 11 & 15 & 26 \\
\hline Asistente Social & 09 & 14 & 23 \\
\hline Matrona & 05 & 10 & 15 \\
\hline Profesor educación física & 09 & 04 & 13 \\
\hline \multirow{2}{*}{$\begin{array}{l}\text { Otros: Educadora de párvulos, médicos, } \\
\text { terapeutas ocupacionales e ingenieros }\end{array}$} & 06 & 08 & 14 \\
\hline & 124 & 188 & 312 \\
\hline
\end{tabular}

\section{CUADRO 1}

Modelo de las Etapas de Cambio.

Etapas

Precontemplación

Contemplación

Preparación

Acción

Mantención

\section{Característica}

La persona desconoce que ciertas conductas lo ponen en riesgo o bien conoce la existencia de un problema de salud pero no tiene ninguna intención de realizar cambios en su conducta.

La persona advierte que ciertas conductas ponen en riesgo su salud o bien se da cuenta de la existencia de un problema de salud, con mayor preocupación por el tema, realizando un análisis activo de los pro y contra. Por todo esto comienza a considerar la posibilidad de cambiar.

La persona piensa seriamente en modificar su conducta en un futuro próximo, el cambio se convierte en prioridad y está listo para tomar decisiones y realizar planes de cambio.

Realiza algunas acciones para que ocurra el cambio.

La persona se encuentra trabajando activamente en las modificaciones de las conductas que atañen a su salud, o bien en el problema de salud identificado. Las estrategias de cambio se eligen y se siguen con una modificación activa de la conducta.

La persona intenta sostener en el tiempo la nueva conducta adquirida, trabajando para evitar las recaídas. Es la etapa que va más allá de los de 6 meses del cambio de conducta. 
que considera la consejería breve en tabaco para embarazadas y población mayor de 15 años, (17,18). Esta inclusión de la consejería en la política de garantías explicitas en salud, es una señal de su valoración en las actividades de salud, pero ha contado con una baja cobertura desde su creación el año 2005.

EI MINSAL reconoce la importancia de las consejerías incentivando su uso y requiriendo el registro del número de consejerías que realizan los profesionales de salud en los establecimientos del sistema público. Sin embargo, se carece de un consenso técnico entre los programas, de aplicaciones o medidas y de un sistema de formación o incentivos al desarrollo de competencias.

\section{Definición de Consejería en Salud}

Es una atención sistemática, basada en la comunicación efectiva, que reconoce en el usuario el papel de protagonista de la adopción de conductas saludables y autocuidado, actuando el profesional como facilitador del proceso de toma de conciencia, decisión y actuación de la persona. Es una intervención comportamental con enfoque psicosocial, que se puede realizar en distintas modalidades (individual/grupal, breve/integral). El consejo breve es la actividad que se realiza dentro de cualquier atención de salud, con una duración de 3 a 5 minutos; y el consejo integral o intensivo es una atención programada de 15 a 20 minutos por cuatro o más sesiones realizadas por un profesional de la salud.

El concepto de consejería en vida sana por lo general se refiere al apoyo para la adopción de conductas saludables en alimentación y actividad física, especialmente consumo diario de frutas y verduras y actividad física regular a la semana, en coherencia con las Guías Alimentarias y de Actividad Física para la población chilena (19-21).

En cambio la consejería en tabaco, incentiva el desarrollo de capacidades para dejar de fumar y mantención de ambientes libres del humo de tabaco, (22).

La guía para el REM define la consejería como una "relación de ayuda, entrega de información y educación que puede realizarse en una o más sesiones, que se desarrolla en un espacio de confidencialidad, escucha activa, acogida y diálogo abierto. Considera las necesidades y problemáticas particulares de cada persona y tiene como objetivo promover y fortalecer el desarrollo de las potencialidades, de manera que la persona descubra y ponga en práctica sus recursos, tome decisiones en la consecución de su estado de bienestar integral. Está dirigida a la población general y debe realizarse con enfoque de derechos, género y no discriminación, con pertinencia cultural y centrada en las necesidades de la persona" (13).

\section{BASES TEORICAS DE LA CONSEJERIA}

Diversas teorías describen el proceso que recorre una persona desde que reconoce la necesidad de un cambio hasta que lo consolida. Se sabe que para que las personas cambien sus conductas el conocimiento no es suficiente; se deben considerar las motivaciones, percepciones, habilidades y condiciones sociales.

La Teoría de Aprendizaje Social o Teoría Social Cognitiva y las teorías de Organización Comunitaria y Ecológica junto a diferentes modelos (Creencias en Salud, Precede/Procede y Procesamiento de la Información por el Consumidor); han contribuido al desarrollo conceptual de la Consejería con propuestas operativas $(2,23,24)$.

Esta base teórica y metodológica ha mostrado su utilidad, mediante estudios de evidencias, especialmente en el ámbito de tabaco, contribuyendo a su aplicación más reciente en alimentación, actividad física y otros componentes de estilos de vida. Han logrado resultados satisfactorios, cuando la consejería se realiza en forma sistemática y los profesionales cuentan con habilidades básicas para su desempeño (25). Actualmente, las aplicaciones más desarrolladas y recomendadas con un uso complementario son el Modelo de Etapas de Cambio (MEC), la Entrevista Motivacional (EM) y la Estrategia de las $5 A$ y $5 R$, las que a continuación se describen.

\section{Modelo de las Etapas de Cambio}

El MEC o Modelo Transteórico de la Etapas Cambio (26) plantea que las personas que logran cambios intencionales de sus conductas habituales, lo hacen a través de un proceso dinámico integrado por etapas, cada una de ellas con características propias más o menos marcadas.

Es un modelo global, aplicable a diversas conductas adictivas y de auto cuidado. Da cuenta de los procesos de cambio identificando los diferentes niveles de predisposición (etapas de cambio) que puede mostrar una persona cuando se plantea modificar su conducta problema. Estas etapas son: precontemplación, contemplación, preparación, acción y mantención; las que se describen en el cuadro 1. En las etapas de acción y mantención pueden ocurrir recaídas, es decir el individuo vuelve a la conducta no deseada. Al iniciar un cambio difícil, la recaída suele ser la regla por lo que, lejos de tomarlo como un fracaso, debe ser interpretado como parte del proceso de cambio, sirven como oportunidades de aprendizaje y es el camino hacia la mantención.

El paso por estas etapas no es lineal, puede haber avances y retrocesos e incluso saltarse etapas. El rol del profesional de la salud es ayudar a la persona, sin presionar, a moverse de una etapa a otra, avanzando de acuerdo a la situación particular de cada uno.

\section{Entrevista motivacional}

La EM es un tipo de intervención clínica centrada en la persona, que ayuda a explorar y resolver ambivalencias sobre una conducta, para promover cambios hacia estilos de vida más saludables. (27-29)

La ambivalencia es la percepción de querer y no querer generar el cambio al mismo tiempo o querer cosas incompatibles al mismo tiempo. Es una fase natural en el proceso de cambio y se considera un aspecto normal de la naturaleza humana, no patológico. La ambivalencia es una cuestión clave a resolver para que se produzca el cambio en las personas.

La EM se aplica especialmente cuando las personas no están dispuestas a hacer un intento por cambiar. Esto puede ser porque: carecen de información acerca de los daños de su conducta o de los beneficios del cambio, tienen algunos miedos o preocupaciones acerca del cambio o están desmoralizados por fracasos previos. La EM intenta ayudar a la persona a desarrollar sus propias habilidades, con vista a estimular el cambio de conducta. Sus componentes son:

- Colaboración: sujeto y consejero trabajan en conjunto. Ambos son "expertos" y se respetan. Al crear una atmósfera positiva, favorece el cambio.

- Evocación: consiste en extraer ideas y soluciones de las personas, después se analiza los caminos que la persona podría elegir.

- Autonomía: la persona es el responsable último en elegir el camino. Esto es desafiante para los profesionales de salud, ya que implica no forzar el cambio.

El objetivo central de la EM es aumentar la motivación intrínseca de la persona, de manera que el cambio surja des- 
de dentro, más que se imponga desde fuera. Para conseguir estos objetivos, utiliza cuatro herramientas: expresar empatía, desarrollar la discrepancia, trabajar la resistencia y apoyar la autoeficacia; cuadro 2.

La EM es una herramienta útil para cambiar conductas de riesgo, con una filosofía centrada en el potencial de cambio de la persona; es tarea del consejero liberar ese potencial para facilitar el proceso de cambio. No juzga y tiene la actitud de ayudar a las personas a ser más abiertos sobre sus pros y contras, focalizándose en la etapa que está casa uno. Se debe dejar de lado la presunción de que el consejero ha fallado si las personas no toman decisiones de cambio en cada atención.

Estrategia de las $5 \mathrm{~A}$ y $5 \mathrm{R}$

Basados en el MEC y la EM se han formulado estrategias de apoyo práctico a personas en proceso de cambio de conductas. La más utilizada es la estrategia de las 5A y las 5R; recomendada por la OMS y las principales guías clínicas de tabaco a nivel internacional $(22,30)$. Si esta estrategia se realiza en forma sistemática, se logra que muchos fumadores se motiven y concreticen intentos por dejar de fumar; estrategias incorporadas últimamente en el manejo de la obesidad.

La estrategia de las 5A se denomina así por la inicial de los 5 pasos que la constituyen: Averiguar, Aconsejar, Acordar, Ayudar y Acompañar. A su vez, las 5R corresponden a la inicial de sus 5 componentes: Riesgos, Relevancia, Recompensas, Resistencias y Repetición, (cuadro 3).

Las $5 \mathrm{~A}$ consisten básicamente en estimular y promover la intención de cambio y ayudar a aquellos ya motivados en dejar el tabaco o cambiar sus conductas de alimentación y actividad física. Las 5R son para aumentar la motivación de personas que no están listas para tomar la decisión de cambio

Con fines operacionales, algunos autores han realizado adaptaciones de esta metodología para tabaco, entre las cuales la estrategia $A B C$ es recomendada por los ministerios de salud de Nueva Zelandia, Uruguay y Chile (9). ABC corresponde a: Averiguar, Breve consejo, Cesación; otras variaciones son: AAA (Averiguar, Aconsejar, Actuar); o bien AAR (Averiguar, Aconsejar, Referir).

El uso combinado del MEC, de la EM y la estrategia de las $5 A$ y $5 R$, constituye hoy día una guía práctica para efectuar consejería en tabaco, alimentación saludable y actividad física y también para la consejería nutricional realizada con fines de tratamiento en personas con sobrepeso y obesidad.

\section{CONSEJERIA Y EVIDENCIA}

La evidencia científica en consejería es abundante, especialmente en tabaco, siendo fuertemente recomendada su implementación. Si bien existen menos evidencias en alimentación y actividad física, hay razones suficientes para su recomendación en función de la existencia de contenidos definidos y repetibles que disminuyen la variabilidad de su aplicación y del efecto logrado en diferentes experiencias evaluadas $(31,32)$.

En la planificación de toda consejería, es preciso tener en cuenta el contexto y la aplicabilidad en diferentes escenarios, así como quienes la desempeñan y los resultados esperados.

\section{Consejería en tabaco}

La literatura muestra que la consejería breve incrementa la abstinencia evaluada a seis meses, de $2.1 \%$ a $5.1 \%$ (33). La consejería integral aumenta la cesación en $6,1 \%$ frente al con-

\section{CUADRO 2}

Herramientas de la entrevista motivacional aplicadas a la Consejería de Alimentación, Actividad Física y Tabaco.

Expresar empatía

Desarrollar discrepancia

Lidiar con la resistencia

Apoyar la autoeficacia
Usar preguntas abiertas para explorar la importancia de la conducta problema, preocupaciones y beneficios de su cambio. Ej.¿Qué ocurriría si usted dejara de fumar?

Escucha activa para manifestar comprensión, resumir y reformular frases.

Ej. Así que usted cree que fumar le ayuda a mantener su peso.

Normalizar sentimientos y preocupaciones.

Ej. Mucha gente se preocupa sobre su sedentarismo.

Apoyar la autonomía de la persona y el derecho a rechazar el cambio.

Ej. usted dice que no está listo para comer 5 verduras y frutas al día.

Destacar la discrepancia entre la conducta actual del sujeto y sus prioridades, valores o metas. Ej. Parece que usted es muy apegado a su familia. ¿Cómo cree que afecta a sus hijos al seguir fumando?

Construir y profundizar en el compromiso al cambio.

Ej. Nos gustaría ayudarlo a evitar un infarto, como el que sufrió su padre.

Retroceda y use reformulación cuando la persona exprese resistencia.

Ej. Parece que se siente presionado para hacer más ejercicio.

Pida permiso para entregar información.

Ej. ¿Le gustaría saber algunas estrategias que pueden ayudarlo a dejar de fumar?

Ayude a la persona a identificar y construir sobre éxitos anteriores.

Ej. Así que le fue bien la última vez que intentó dejar de fumar.

Ofrezca opciones para lograr pequeños pasos hacia el cambio.

Ej. Empiece a caminar más bajándose un paradero antes del metro o el microbús. 
sejo breve. Además, el tiempo total de contacto -número de sesiones y duración - incrementa la cesación en $17.4 \%$ hasta los 300 minutos, sin beneficio adicional después de este tiempo; el número de sesiones incrementa la cesación hasta en $12.3 \%$.

La consejería en tabaco realizada por médicos, enfermeras y dentistas tiene mayor efectividad que la realizada por otros profesionales. El consejo breve de un médico de APS durante una consulta de rutina aumenta en forma efectiva el número de fumadores que dejan de fumar por lo menos por 6 meses. (34).

La consejería realizada por enfermeras es efectiva. En una revisión con más de 17.000 fumadores, la intervención de enfermeras aumentó la probabilidad de cesación (35). La consejería realizada por dentistas a fumadores, aumenta las tasas de abstinencia a 6 meses o más. En una revisión de 14 ensayos clínicos con más de 10.500 participantes, se encontró un OR de 1.71, IC 95\% 1.44 a 2.03 (36).

Respecto del formato de la consejería en tabaco, se puede realizar en forma presencial (cara a cara), individual o grupal, y por teléfono e internet. La consejería grupal no tendría diferencias con la consejería individual, aunque se sugiere que sería más costo-efectiva (30).

El consejo telefónico reactivo (una única llamada) no ha mostrado beneficios, pero la consejería telefónica proactiva (con seguimiento) es efectiva. Agregar consejería telefónica a la consejería cara a cara tiene mayor eficacia que esta última

\section{CUADRO 3}

Estrategia de las 5A y 5R en la Consejería de Alimentación, Actividad Física y Tabaco.

Primera A

Averiguar.

Segunda A Aconsejar:

Tercera A Acordar el tipo de intervención

Cuarta A

Ayudar.

Quinta A.

Acompañar.

Primera R

Riesgos:

Segunda $R$

Relevancia:

Tercera R

Recompensas:

Cuarta R

Resistencias

Quinta R

Repetición:
Preguntar a todas las personas por las conductas de salud aprovechando todas las instancias por las cuales concurren a los establecimientos de salud. Ej: cantidad de ejercicio, consumo de frutas y verduras, si fuma o no.

Aconsejar a la persona para que modifique la conducta problema. El consejo debe ser claro y firme, personalizando los argumentos de convencimiento. Ej:

Claro: Controlar su peso es muy relevante para disminuir su riesgo de diabetes

Fuerte: Alimentarse en forma sana, es algo importante que usted puede hacer para proteger su salud Personalizada: "Ahora que está embarazada..." "Su hijo corre riesgo de .... por...."

Existen diferentes tipos de intervención, dependiendo de la etapa de cambio en que se encuentre. -Si la persona no desea cambiar su conducta ahora (etapa de precontemplación y contemplación): realizar una intervención motivacional, aplicando las 5R.

-Si la persona quiere cambiar la conducta y está decidido (etapa de preparación): ofrecer ayuda y planear una estrategia para el cambio.

Si la persona está dispuesta a intentar el cambio, ayudar para desarrollar un plan de acción:

- En tabaco implica fijar una fecha para detener el consumo (día D) y proporcionar consejos prácticos

- En actividad física, ver posibilidades de incorporar caminatas u otra actividad en su vida cotidiana.

- En alimentación revisar por ejemplo las posibilidades reales de consumir más frutas y verduras.

En las personas que están dispuestas a cambiar su conducta, se debe programar el seguimiento, que puede ser cara a cara o telefónico.

Ayudar a que la persona identifique los riesgos de la conducta problema con algunas preguntas útiles: ¿Qué sabe usted acerca de los riesgos para la salud de fumar? ¿Qué es lo que más le preocupa a usted? Ud. se precia de tener buena salud: ¿Cómo será en el futuro si no cuida su peso?

Discutir con la persona la importancia que tendría cambiar la conducta problema. Ej: ¿Cuán importante es para usted dejar de fumar? ¿Cuáles serían los aspectos más positivos de bajar de peso para Ud.? ¿Cómo beneficiaría a su familia si Ud. deja de fumar?

Incentivar a la persona a que reconozca los beneficios del cambio de conducta.

Ej: ¿Cuáles serían los beneficios de dejar de fumar para usted? especialmente en cuanto al riesgo de cáncer que a usted le preocupa. ¿Cómo se sentiría si bajara de peso?

Intentar que la persona identifique las barreras para cambiar su conducta y ofrecer ayuda para enfrentarlas, como temor a los síntomas de abstinencia, aumento de peso u otra. Ejemplo: ¿Cuáles serían las principales dificultades de dejar de fumar para usted? Parece que se siente presionado a cambiar su dieta? ¿Así que usted cree que fumar le ayuda a mantener su peso?

Repetir la evaluación de si está listo para el cambio. Si no está listo, repetir la intervención en una próxima oportunidad. Ej: Lo escucho decir que usted no está listo para hacer un cambio ahora.

Estaré aquí para ayudarla cuando si lo esté. Será fantástico que intente dejar de fumar cuando pase este momento complicado en su trabajo. 
sola. Hay evidencias de una relación dosis-respuesta en cuanto al número de llamadas y tasas de cesación, lográndose los mejores resultados entre 3 y 6 llamadas (37).

Las intervenciones por internet tienen un beneficio menor que las intervenciones personalizadas OR 1.3 IC95\% 1.2-1.3. Sin embargo los resultados no son consistentes (38).

La consejería para la cesación del tabaquismo tiene una amplia difusión en la literatura médica. Es eficaz y las guías clínicas recomiendan utilizarla frente a todos los fumadores, la que puede ser realizado por distintos profesionales de la salud. La consejería integral se puede ofrecer en forma individual, grupal o telefónica de manera proactiva.

Si bien hay menos estudios sobre la eficacia de la consejería para mantener ambientes libres del humo de tabaco se recomienda su uso por los beneficios en disminuir los riesgos del fumar pasivo, en especial en grupos que requieren mayor protección ambiental, adultos mayores, niños y embarazadas. (39). El consejo en tabaco aumenta aún más su efectividad, cuando es complementada con medidas estructurales, como son el aumento de los impuestos, la regulación de la publicidad y prohibición de venta a menores, entre otros aspectos contenidos en la legislación del tabaco.

\section{Consejería en actividad física}

El análisis de evidencias muestra que los programas individuales de consejería para incentivar la actividad física en adultos son recomendados, con evidencia suficiente para incentivar la práctica de la actividad física asociada a la vida cotidiana de las personas (caminar, bailar, evitar uso de ascensores, andar en bicicleta).

La evidencia sugiere que el ejercicio prescrito por el médico tratante u otro profesional de salud, aumentaría la tasa de inicio así como la adherencia a este cambio en el estilo de vida (40-42).

Para niños y adolescentes menores de 18 años, las intervenciones más efectivas se desarrollan en el ámbito escolar, con colaboración de la familia; la consejería que se realiza en forma aislada no logra un aumento significativo de la actividad física. El consejo en personas sanas aumenta la actividad física a corto plazo (4 a 6 meses), pero son menos los estudios que lo demuestran a largo plazo, (24).

\section{Consejería alimentación}

Es más limitada, puede producir pequeños a moderados cambios en el consumo promedio diario de frutas y verduras, y de grasas saturadas. Existe recomendación de evidencia para el uso de consejería integral en la prevención de las ECNT con grupos de población seleccionados; en cambio para la población general la evidencia es insuficiente $(43,44)$.

Otras investigaciones muestran un efecto favorable de esta consejería en grupos con factores de riesgo (45-47); hallazgos que sugieren el desarrollo de nuevos estudios, ampliando las poblaciones estudiadas.

La consejería en actividad física y alimentación, aun cuando no cuenta con una evidencia fuertemente recomendada sobre su eficacia en población general, se recomienda por los beneficios en la reducción del riesgo y las mejoras en la calidad de vida relacionada con la salud. En grupos de riesgo, cuando son realizadas por nutricionistas, profesores de educación física y kinesiólogos tienen mayor efectividad que la realizada por otros profesionales.

Para lograr mayor efectividad y eficacia, estas intervenciones conductuales requieren ser multidisciplinarias, complementarse con medidas estructurales y una acción intersectorial sostenida, como: la obligatoriedad y aumento de horas de actividad física en los colegios, restricciones a la publicidad y venta de alimentos no saludables, acceso a frutas y verduras, oferta de preparaciones saludables, pausas activas en el ambiente laboral, disponibilidad de ciclovías, parques y otros medios para la práctica de la actividad física.

La investigación señala que intervenciones poblacionales con multiestrategias continuas en el tiempo (consejería, educación, comunicación, participación social y política pública), presentan mejores resultados que intervenciones separadas $\mathrm{o}$ aisladas.

\section{LA PRÁCTICA DE LOS PROFESIONALES DE LA APS}

Se presentan aspectos relativos a la experiencia observada en los participantes de los programas de capacitación del INTA (cuadro 1). El 85\% señala tener experiencia en consejería, reconociéndola como una actividad regular de sus funciones durante el último año de trabajo, en general registrada e informada y que le destinan un tiempo importante de su jornada laboral. Sin embargo, se trata de una atención individual realizada con diferentes enfoques, sin evaluación ni retroalimentación a su práctica.

Las consejerías que realizan con mayor frecuencia son en alimentación saludable y actividad física, alcohol y drogas, lactancia materna, tabaco, salud mental, salud oral, salud sexual y en $\mathrm{VIH}$.

Un tercio de los profesionales realiza consejo breve en los controles de salud y en los Programas de Salud Cardiovascular, de la Mujer y del Adulto Mayor; predominando en ellos una transmisión de mensajes de la vida saludable, sin continuidad o refuerzo en controles siguientes. Otros refieren realizar la consejería para aumentar la adherencia de los usuarios o mejorar la calidad de atención. Les preocupa no tener criterios estandarizados de aplicación y la exigencia de cantidad sobre calidad, su motivación para aprender radica en el interés de tener mayores logros y satisfacciones.

Reconocen poseer condiciones personales para realizar consejería, mencionando con mayor frecuencia: a) Empatía y motivación. b) Capacidad de escucha, paciencia y flexibilidad. c) Habilidades comunicacionales, buen trato, experiencia en educación y conocimiento de su realidad. Se observa coincidencia en la percepción de las dificultades que enfrentan para realizar consejería, siendo mayormente: a) Falta de tiempo protegido. b) Falta de interés de los usuarios, inasistencia, vulnerabilidad social. c) Frustración por no lograr cambios de conducta y escaso conocimientos y herramientas de apoyo para realizar la consejería.

Las condiciones laborales para realizar consejería, varían entre los establecimientos de salud según el enfoque de atención, los recursos disponibles y la iniciativa de los liderazgos locales. El cuadro 4, muestra la percepción de obstáculos y facilitadores para realizar consejería en la APS.

Al término de la capacitación los participantes realizan un análisis de oportunidades para realizar una consejería de mayor calidad y efectividad (cuadro 5). Destacan el potencial de nuevos programas, la certificación en la implementación del modelo de salud familiar en los Centros de Salud Familiar, y la posibilidad de incorporar la consejería en la programación anual de actividades de salud municipal, con metas más realistas conforme a la evidencia. Para el mejoramiento de la consejería plantean la necesidad de elaborar protocolos de atención individual y grupal, con orientaciones para mejorar el flujo de atención.

Existe unanimidad en reconocer la actividad de consejería 
como una herramienta fundamental en la práctica de salud, que todo profesional y técnico que atiende personas debiera poseer.

\section{DISCUSION}

La integración de actividades preventivas ha sido asumida tanto por las instituciones prestadoras de salud, como por profesionales. Sin embargo, en la consejería, se da una paradoja, ya que se encuentra recomendada en las políticas y programas, con escaso acompañamiento para su implementación, observando una brecha entre la teoría y la práctica.

Hay quienes justifican este actuar por una menor evidencia científica, sin reconocer que la existente es suficiente y que además de los resultados de investigaciones científicas de alta calidad, se cuenta con evidencia sobre los efectos de las intervenciones y su mayor relevancia en función del perfil epidemiológico del país, caracterizado por alta prevalencia de obesidad, tabaquismo y ECNT. Otra corriente de influencia es la subvaloración de las intervenciones psicosociales ante las acciones de política estructural apoyadas en el enfoque de determinantes sociales de la salud, desconociendo la necesaria complementariedad de las disciplinas (48). No tomar acciones para el mejoramiento de esta actividad de salud, ha de justificarse solo desde criterios políticos y organizativos, pero no en niveles de evidencia insuficiente.

Los profesionales de la salud realizan consejería en forma cotidiana, la que frecuentemente no logra sus objetivos. Otro factor que influye, es la falta de formación en consejería en un contexto que valora mayormente la formación clínica en desmedro de la prevención y promoción de salud. La experien-

\section{CUADRO 4}

Obstáculos y facilitadores para realizar Consejería en la APS percibidos por profesionales de salud.

\section{Obstáculos}

Facilitadores
-Alta carga laboral con exigencia de metas asistenciales, sin tiempo protegido para consejería.

- La consejería en alimentación no está considerada en las prestaciones de salud.

- Consejería breve dentro de una atención, no es reconocida en estadísticas.

-Falta de recursos humanos y de box de atención.

- Funcionarios no respetan privacidad en la consejería, falta de capacitación.

- Usuarios con escasa motivación y baja adherencia, vulnerabilidad social.
-Modelo salud familiar con equipos multi- disciplinarios que conocen a su población

-Profesionales con flexibilidad para realizar consejería: enfermera en control de salud, asistente social en programa psicosocial, psicólogo con nutricionista en ingreso de diabéticos.

- Consejería tabaco reconocida como función de kinesiólogos y dentistas.

- Estrategias de derivación interna en la APS y trabajo intersectorial local.

- Población rural con mayor disposición.

- Algunos equipos capacitados cuentan con habilidades y herramientas, motivación en otros para aprender.

\section{CUADRO 5}

Oportunidades y recomendaciones para la Consejería en la APS.

- Incorporar el consejo breve en forma sistemática en toda consulta nutricional, en los controles de salud y en las visitas domiciliarias.

- Realizar el consejo breve según protocolo en el Examen de Medicina Preventiva del Adulto y del Adulto Mayor (EMPA y EMPAM).

- Mejorar rendimiento de actividades grupales programadas, incorporando la consejería. Por ejemplo en talleres de lactancia materna y talleres de actividad física.

- Incluir la consejería grupal en el trabajo con escuelas y jardines infantiles, con grupos de autoayuda y organizaciones sociales.

- Realizar consejería en las ferias de salud y otras actividades comunitarias de promoción de salud.

- Programar la consejería con criterios comunes y guías de derivación en base a la sectorización de los equipos de salud familiar.

- Programas de Salud con oportunidades para realizar consejería: Programa Vida Sana, Programa de Salud Adulto Mayor, Programa Más Adultos Mayores Autovalentes, Programa del Adolescente y Programa de Salud Cardiovascular. 
cia que reportan los equipos de salud, analizada con nuevos conocimientos, abre un potencial de desarrollo para la APS.

Si bien existe evidencia sobre los mejores resultados alcanzados por algunos profesionales en la consejería de alimentación, actividad física y tabaco, la realidad del país en términos de disponibilidad de recursos humanos en salud y nutrición, permite plantear que cualquier profesional entrenado puede realizar una actividad de consejería de calidad que aporte a las metas de salud.

En nuestra experiencia, los profesionales más interesados en la formación en consejería han sido nutricionistas, kinesiólogos y odontólogos, con escasa demanda por parte de los médicos. Esta realidad da cuenta que los contenidos curriculares del pregrado en educación médica, no incorporan el desarrollo de estas habilidades. Recientemente, la Asociación Americana del Corazón, ha emitido un consenso científico para el desarrollo de competencias en consejería en estilos de vida, como práctica esencial en la formación médica para la prevención y tratamiento de los ECNT (49).

El INTA ha extendido esta formación a técnicos, obteniendo resultados muy satisfactorios en términos de cobertura y rendimiento académico (3).

\section{CONCLUSIÓN}

Esta actualización teórica y metodológica de la consejería, con propuestas para el mejoramiento de la práctica de los profesionales de salud y nutrición, puede aportar a establecer un consenso técnico y adoptar medidas concretas que permitan fortalecer una política pública para el alarmante aumento de la obesidad y de las ECNT en el país.

\section{RESUMEN}

El objetivo de este artículo es presentar una actualización de los fundamentos teóricos y metodológicos de la consejería y la evidencia sobre su eficacia, relacionándola con la práctica de los equipos de salud y analizando los desafíos futuros. Se describen las bases teóricas, las orientaciones de política del Ministerio de Salud de Chile, las estrategias recomendadas a nivel internacional y los aportes de los participantes de un programa de capacitación realizado por el INTA con 312 profesionales (nutricionistas, kinesiólogos y odontólogos en su mayoría). Se presenta el análisis de las condiciones en la Atención Primaria de Salud para implementar la consejería en alimentación, actividad física y tabaco en forma sistemática. Se discute la necesidad de adoptar medidas para el mejoramiento de la práctica y su contribución a un abordaje preventivo de los graves problemas de sedentarismo, obesidad y tabaquismo en la población chilena.

Palabras clave: consejería en alimentación, actividad física, tabaco, etapas de cambio, entrevista motivacional, estrategia $5 A$ y $5 R$

\section{BIBLIOGRAFÍA}

1. Vio F, Albala C, Kain J. Nutrition transition in Chile revisited: mid-term evaluation of obesity goals for the period 2000-2010. Public Health Nutr 2008; 11(4):405-12.

2. Sánchez $H$, Moreno $X$, Bustos N. Promoción de estilos de vida saludables en Centros de Atención Primaria En: Albala C, Olivares S, Salinas J, Vio F. eds Bases, prioridades y desafíos de la promoción de la Salud Impresión Andros, Chile 2004 p 228-39.

3. Salinas J, Muñoz C, Albagli A, Araya G, Vio F. The contribution of distance education to health promotion in Chile. Health Promot Int. Advance Access published Apr 22, 2016; doi:10.1093/heapro/daw023.

4. Salinas J, Donoso N, Molina H, Montecinos C, Pezoa D, Torres M. Plan Nacional de Promoción de Salud. Santiago: Mosquito Comunicaciones; 1999.

5. Salinas J. Reorientación de Servicios de Salud con criterios de Promoción de la Salud. Rev Chil Med Fam 2002; 3 (2): 84-95.

6. Albala $C$, Olivares $S$, Sánchez $H$, Bustos $N$, Moreno X, Bravo C, Barraza A., Consejería en Vida Sana. Manual de Apoyo. INTA-MINSAL, 2004.

7. Albala C, Olivares S, Sánchez $H$, Bustos $N$, Moreno X, Consejería Breve en Vida Sana. Manual de Apoyo. INTAMINSAL, 2004.

8. Castro R, Gonzalez M, Lopez C. eds Manual de Atención Personalizada en el Proceso Reproductivo. División Prevención y Control de Enfermedades. MINSAL 2008.

9. Paccot M, Escobar MC, Rozas MF, Srur A y cols Enfoque de riesgo para la prevención de enfermedades cardiovasculares. MINSAL. Consenso 2014.

10. Escobar MC, Olivares S, Zacarías I. Manejo alimentario del adulto con sobrepeso y obesidad. Programa de Salud Cardiovascular. MINSAL, OPS/OMS, Chile 2002

11. Acuña $M$ y cols La cesación del consumo de tabaco. Programa de Salud Cardiovascular. MINSAL, OPS/OMS, Chile 2003.

12. Escobar MC, Zacarías I. eds. Programa de Actividad Física para la prevención y control de los factores de riesgo cardiovascular. Programa de Salud Cardiovascular MINSAL, OPS/OMS, Santiago Chile 2004.

13. Ministerio de Salud. Departamento de Estadísticas e Información DEIS. Manual de Instrucciones Series REM 20142016 Resumen Estadístico Mensual REM-A.19a Actividades de Promoción, Prevención y Participación. 2014.

14. Dois A, Ojeda I, Vargas I, Larrea M, Quiroz M, et al Orientaciones para la Implementación del Modelo de Atención Integral de Salud Familiar y Comunitaria. División de Atención Primaria. MINSAL 2013.

15. Ministerio de Salud. Subsecretaría de Salud Pública. Orientaciones para la Programación en Red. Chile 2016.

16. Ministerio de Salud. Subsecretaría de Redes Asistenciales. División de Atención Primaria. Instrumento para la Evaluación y Certificación de Desarrollo en el Modelo de Atención Integral de Salud Familiar y Comunitaria en Establecimientos de Atención Primaria. Chile 2015.

17. Ministerio de Salud, Subsecretaría de Salud Pública, Guía Clínica Examen Medicina Preventiva. 2008.

18. Ministerio de Salud. Manual de Aplicación del Examen de Medicina Preventiva del Adulto Mayor. Programa de Salud del Adulto Mayor. 2010.

19. Ministerio de Salud. Guías alimentarias para la población. Chile 2013.

20. Vio F, Salinas J., eds. Guía para una vida activa. Universidad de Chile, INTA; MINEDUC; MINSAL; Consejo VIDA CHILE; Chile Deportes; 2003 Andros 2003.

21. MINSAL Chile, Norma General Técnica N`76 Guía Educativa para una Vida Saludable. Guías Alimentarias, Actividad Física y Tabaco. Resol Ex No 459 del 20.08.2005.

22. Bello S, Flores A, Bello M, Chamorro H. Diagnóstico y tratamiento psico-social del tabaquismo. Rev Chil Enf Respir 2009; 25: 218-230.

23. La aplicación de teorías y técnicas de las ciencias sociales a la promoción de la salud Rev Panam Salud Publica/Pan Am J Public Health 4(2), 1998.

24. Depaux R. Acciones efectivas sobre cambio en estilos de 
vida. Rev Chil Med Fam. 2011; 11(1):29-39.

25. Public Health Agency of Canada. Motivational Interviewing. Videos on supporting Behaviour Change Disponible en http://www.phac-aspc.gc.ca/cd-mc/videos/index-eng.php Consultado el 07/07/2016

26. Prochaska J, Diclemente C. Stages and processes of selfchange of smoking: toward an integrative model of change. J Consult Clin Psychol. 1983; 51: 390-95.

27. Lizarraga S, Ayarra M. Entrevista motivacional ANALES Sis San Navarra 2001; 24 (Supl. 2): 43-53.

28. Rivera S, Villouta F., llabaca A., Entrevista motivacional: ¿Cuál es su efectividad en problemas prevalentes de la atención primaria? Aten Primaria. 2008; 40(5):257-61

29. Miller W, Rollnick S. Motivational Interviewing: Preparing people for change. 2. ${ }^{a}$ eds. New York: Guilford Press; 2002.

30. U. S, Preventive Services Task Force Recommendation Statement. Behavioral and Phamacotherapy Interventions for Tobacco Smoking Cessation in Adults, including Pregnant Women. Ann Intern Med. 2015; 163(8): I-40. doi: 10.7326 / P15-9032.

31. Kraemer P, Escobar MC. Eds Revisión sistemática de evidencia científica sobre intervenciones individuales para apoyar a los fumadores a dejar el hábito de fumar MINSAL 2012.

32. Nebot M. El consejo médico y la promoción de salud. Atención Primaria 1991; 8(4): 19-23.

33. Alba L, Murillo R, Castillo JS. Intervenciones de consejería para la cesación de la adicción al tabaco: revisión sistemática de la literatura. Salud Publica México 2013; 55 (2): 196-206.

34. Stead LF, Buitrago D, Preciado N, Sanchez $G$, HartmannBoyce J, Lancaster T. Physician advise for smoking cessation (review) Cochrane Database of Systematic Reviews 2013, Issue 5. Art. $N^{\circ}$ CD000165. DOI: 10.1002/14651858. CD000165.pub4.

35. Rice VH, Hartmann-Boyce J, Stead LF. Nursing interventions for smoking cessation (review). Cochrane Database of Systematic Reviews 2013, Issue 8. Art. N: CD001188. DOI: 10.1002/14651858. CD001188.pub4.

36. Carr $A B$, Ebbert J. Interventions for tobacco cessation in the dental setting (review). Cochrane Database of Systematic Reviews 2012, Issue 6. Art. No: CD005084. DOI: 10.1002/14651858.CD005084.pub3.

37. Fiore M.C, Jaén C. R., Baker T.B., Bailey W.C., Benowitz $N$, Curry S.J. et al. Treating Tobacco Use and Dependence: 2008 Update. Clinical Practice Guideline. Rockville, MD: US Department of Health and Human Services, Public
Health Service; 2008.

38. Civljak M, Scheikh A, Stead LF, Car J. Internet-based interventions for smoking cessation. Cochrane Database of Systematic Reviews 2010, Issue 9. Art. N: CD007078.

39. WHO. Toolkit for delivering the $5 A$ 's and $5 R$ 's brief tobacco interventions in primary care. ISBN 9789240692619 (ePUB) (c) World Health Organization 2014.

40. Ciapponi A. Actualización y combinación de las guías de cuidados preventivos de las fuerzas de tareas de EE.UU. y Canadá.(Primera parte: Consejería). Evid Act Pract Ambul. 2009; 12(2). 61-5.

41. American College of Sports Medicine, Exercise is Medicine, "Evidence for EIM - Physical Activity and Non-Communicable Diseases (NCD's). 2009.

42. Ramírez-Cardona L, Trejo-Varón R; Barengo N. Prácticas y consejería de actividad física en médicos generalistas de Argentina Rev. Salud Pública. 2013, 15 (3): 455-64.

43. U.S. Preventive Services Task Force (USPSTF). The Guide to Clinical Preventive Services. Recommendations of USPSTF 2014.

44. Castillo A, Smith C, Hirsch S, Brito A. ¿Es efectiva la consejería para aumentar el consumo de frutas y verduras $y$ disminuir el riesgo cardiovascular en prevención secundaria? una revisión. Rev Chil Nutr. 2008; 35(2):123-9.

45. Cerezo S, Ríos I, Brito A, De Romaña D, Olivares M, Pizarro $F$. Consejería nutricional incrementa el consumo de alimentos ricos en calcio, pero la ingesta se mantiene por debajo del requierimiento diario. Rev Chil Nutr. 2014; 41(2):131-8.

46. Abrantes $P$, Halpern-Silveira D, Formoso C. Lifestyle counseling reduces metabolic syndrome prevalence in the short term. Rev Chil Nutr. 2013; 40(3): 216-23.

47. Castillo A., Kain J. Consejería en vida sana y cambio de conductas en escolares obesos: intervención controlada en madres/cuidadoras Rev Chil Nutr. 2010, 37: 2.

48. Fuentes A. Aporte de la psicología y sociología a la Promoción de la Salud en Chile. Cuad Med Soc. 2008, 48:2:118-124.

49. Hivert $M F$, Arena $R$, Forman $D$, Kris-Etherton $P, M c B r i d e$ $P$, Pate $R$, Spring B, Trilk J, Van Horn L, Kraus W. Medical Training to Achieve Competency in Lifestyle Counseling: An Essential Foundation for Prevention and Treatment of Cardiovascular Diseases and Other Chronic Medical Conditions A Scientific Statement From the American Heart Association. Circulation 2016;134:00-00. DOI: 10.1161/ CIR.0000000000000442 October 11, 2016. 